The real problem of galaxy formation remain very much unsolved. The greatest difficulty is that we still have no idea what induced the formation of

\title{
How to study the birth of galaxies using radio-astronomical techniques
}

by John Gribbin

IF you accept the standard picture, galaxies and clusters of galaxies were formed by condensation from a more uniform distribution of hydrogen gas. But just how hydrogen clouds transformed into galaxies is far from clear, and there has seemed little chance of making the situation more clear by direct observation. Now, however, Sunyaev and Zel'dovich have come up with a suggestion that radio observations might provide a clue to events occurring at the epoch when galaxies were forming in the Universe (Mon. Not. R. astr. Soc., 171, 375-379; 1975).

Some of the principal questions which are far from being answered are listed by the two Soviet astronomers: Were globular clusters formed first and later joined into galaxies? Did the first step consist of separation and condensation of giant gas clouds (with masses in the range $10^{13}-10^{15} M_{\odot}$ ) which later disintegrated into galaxies, globular clusters and individual stars? At what redshift $z$ (relative to us) did this occur? And what were the density and temperature of the gas clouds from which galaxies formed?

In principle at least, some of these questions might be answered by radioastronomy studies not hopelessly more sophisticated than those of the present day. One approach would be to search for the redshifted $21-\mathrm{cm}$ hydrogen line emission from the epoch just before galaxies were formed.

Sunyaev and Zel'dovich consider the case of spherical galaxies with mass $3 \times 10^{14} M_{\odot}$ and diameter $2 \mathrm{Mpc}$, separated by $30 \mathrm{Mpc}$. Making reasonable assumptions about the proportion of neutral hydrogen, $\mathrm{H} \mathrm{I}$ and $\mathrm{He} \mathrm{I}$, and taking the diameter of a cloud just before stars formed as also being typically $2 \mathrm{Mpc}$, it turns out that the hydrogen line should still be seen as an emission line, even with a redshift of $5-10$, if at least half the protogalactic material was in the form of hot gas $(T \sim 7,000 \mathrm{~K})$. The increase in temperature of the observed line over the background is, however, only expected to be some $7 \mathrm{~K}$, for an observer co-moving with the protogalaxy.

But to an observer on Earth the line will be redshifted to $100-200 \mathrm{~cm}$ wavelength, a region where the overall background has a brightness temperature ranging from 20 to $200 \mathrm{~K}$. Is it sensible to expect to be able to detect the redshifted hydrogen line above this background? The answer depends, of course, on just how many proto-galactic clouds there might be at these large redshifts in each solid angle of sky subtended by the observing telescope. Sunyaev and Zel'dovich conclude that no more than 200 protoclusters might be visible over the whole sky at any one wavelength, and that "observations with a variable wavelength are essential, both in order to augment the number of protoclouds observed and also to distinguish them from sources of different type with continuum spectra".

They also suggest still more complicated experiments, but this one test is likely to be enough to tax the ingenuity of radio astronomers at present. Still, the possible results would. seem to merit some effort being made. As Sunyaev and Zel'dovich say, "these observations are very difficult and substantial improvement of existing equipment will be needed to fulfil the task, but the importance of the problem seems . . . to warrant discussion in order to attract the attention of experimentalists".

- One in a million. Meanwhile, a reminder to galaxy hunters that not all of the peculiarities of unusual astronomical objects result from physical processes hinting at new universal laws, and that coincidences do happen, has come from a study by de Vaucouleurs and de Vaucouleurs of NGC985-a ring galaxy with a Seyfert nucleus (Astrophys. J. Lett., 197, L1; 1975).

If ring galaxies do form when normal galaxies collide with intergalactic clouds (as Binney discusses above), and if, as seems likely, some (and perhaps all) normal galaxies experience Seyfert outbursts, it is plausible that the formation of the ring in NGC985 and the presence of Seyfert activity are completely unrelated. De Vaucouleurs and de Vaucouleurs suggest that the probability of a randomly chosen $\mathrm{Sa}-\mathrm{Sc}$ spiral having encountered an intergalactic gas cloud in the past $5 \times 10^{7}$ yr (so that the ring is still visible) is about $10^{-4}$, and that the probability that the nucleus of a giant spiral is seen today in the Seyfert stage is about $10^{-2}$. That makes NGC985 literally a galaxy in a million. the first bound objects in an expanding Universe. One suspects that the origin of these local inhomogeneities in the structure of the Universe will be understood only when we know why the Universe is so remarkably smooth and homogeneous on a large scale and a major effort is now going into the possibility that this isotropy is the result of quantum effects back in the period when the Compton wavelength of an elementary particle was comparable with the radius of the then visible Universe. The other urgent task in cosmology is to understand how in the more recent past great masses of gas collapsed to form galaxies and clusters of galaxies. This task is particularly exciting because there is every reason to believe that we should be able to actually see this happening by looking out into distant space. What we require are detailed models of the collapse to enable us to identify a nascent galaxy when we see one.

\section{Specific response of Drosophila cells to heat shock}

\section{from Benjamin Lewin}

When larvae of Drosophila melanogaster are subjected to a heat shock, usually a rise in temperature to $37^{\circ} \mathrm{C}$ from the usual $25^{\circ} \mathrm{C}$, a change in the pattern of puffing is seen in the salivary gland chromosomes; preexisting puffs regress and new puffs appear at several, specific sites. The same response is produced by treatment with respiratory inhibitors and occurs during recovery from anaerobiosis. The switch to puffing at these bands presumably represents the expression of new gene functions and, because of the different treatments which elicit it, may perhaps constitute some generalised response to certain environmental abuses of Drosophila larvae. The specific puffing pattern can be provoked by heat shock of isolated salivary glands and so must be autonomous. It is not known what functions it represents; but the limited number of bands that is involved and their coordinate expression in response to more than one treatment of larvae or excised tissues suggest that this may be a useful system for investigating genetic control and defining the number of functions in individual bands.

Two recent series of experiments exploit the remarkable observation that heat-shocked tissue culture cells of Drosophila seem to display the same response as whole larvae or isolated salivary glands. The cultured cells in both sets of experiments are derived from Schneider's S2 line, 